Africa (CANTAM), West Africa (WANETAM), and Southern Africa (TESA).

\section{PO 8289 ASSOCIATION BETWEEN PLASMA LEVELS OF IL-27, IL-6 CYTOKINES AND P. FALCIPARUM INFECTION IN PREGNANT WOMEN LIVING IN MBALMAYO, CAMEROON}

1,2Balotin Fogang* ${ }^{*}$ ' Jean C Djontu, ${ }^{1}$ Rosette Megnekou, ${ }^{2}$ Lawrence Ayong. ${ }^{1}$ University of Yaounde I, Yaounde, Cameroon; ${ }^{2}$ Centre Pasteur du Cameroon, Yaounde, Cameroon

\subsection{6/bmjgh-2019-EDC.68}

Background The appropriate balance between anti-inflammatory and pro-inflammatory cytokines is necessary for protection against pregnancy-associated malaria and poor pregnancy outcomes. This study therefore aims to investigate the relationship between plasma levels of some regulatory cytokines and $P$. falciparum infection in Cameroonian women during pregnancy.

Methods Peripheral blood was collected from 131 women during pregnancy and 27 non-pregnant women living in the Mbalmayo area between May and December 2014. Parasitaemia was determined by microscopy and haemoglobin level using a haematological counter. Plasma levels of IL-27 and IL6 cytokines were measured using the Magnetic Luminex Screening Assay technique.

Results Parasitaemia associated negatively with haemoglobin level $\left(r_{s}=-0.43 ; p<0.001\right)$. The plasma level of IL-6 was higher in pregnant women than in non-pregnant women $(p=0.05)$. Regarding parasitaemia, plasma level of IL-27 was significantly higher in non-infected than in infected women $(p=0.028)$ while that of IL-6 was significantly higher in infected women $(p<0.0001)$. Moreover, parasitaemia correlated negatively with the plasma level of IL-27 $(p=0.034)$ and positively with that of IL-6 $(\mathrm{p}<0.0001)$. In addition, level of IL-6 was significantly higher in anaemia-positive than in anaemia-negative women $(p=0.028)$. On the other hand, level of IL-27 negatively associated with the parity $(p=0.022)$ and gestation age $(\mathrm{p}=0.014)$.

Conclusion These results show that in pregnant women, $P$. falciparum malaria infection is associated with high plasma level of IL-6 and low level of IL-27, suggesting that IL-27 could have a protective effect against pregnancy-associated malaria while IL-6 seem to be a potential biomarker of the disease.

\section{PO 8290 INFLUENCE OF THE SICKLE CELL TRAIT ON PLASMODIUM FALCIPARUM TRANSMISSION IN ASYMPTOMATIC CHILDREN}

${ }^{1}$ Christelle Ngou Maffo*, ${ }^{3}$ Antoine Berry, ${ }^{4}$ Isabelle Morlais, ${ }^{1}$ Lionnel B Feufack Donfack, ${ }^{1}$ Albert Bayibeki Ngano, ${ }^{2}$ Sandrine E Nsango, ${ }^{1}$ Elangwe Milo Sarah-Matio, 'Lawrence Ayong. 'Malaria Unit, Centre Pasteur du Cameroun, Yaounde, Cameroon; ${ }^{2}$ Faculty of Medecine University of Douala, Yaounde, Cameroon; ${ }^{3}$ Service de ParasitologieMycologie CHU Toulouse/Université Toulouse III, France; ${ }^{4}$ Equipe MIVEGEC-IRD de Montpellier, France

\subsection{6/bmjgh-2019-EDC.69}

Background The sickle cell trait is associated with protection against severe malaria. Recently, it has been shown that the genetic protection conferred by the sickle cell trait has no effect on the transmission of Plasmodium species from humans to vectors. Our study aimed to investigate the putative association between the sickle cell trait AS and the susceptibility to malaria infection of both the human host and the insect vector.

Methods The study was conducted from June to November 2017 among asymptomatic children living in Cameroon. The samples were collected on microscopy slides, Whatman FTA and grade 17 paper for the selection of gametocyte carriers by microscopy, the molecular diagnosis of Plasmodium species, and sickle cell trait (PCR- RFLP), respectively. Infectivity of the mosquito was measured by experimental infections on gametocyte-containing blood from naturally infected carrier. Genetic diversity was measured using microsatellite markers. Results A total of 1557 children were recruited; the prevalence of Plasmodium infection among this group was 58\% and the AS sickle cell trait $20 \%$. No significant difference in the prevalence of $P$. falciparum infection was observed according to the sickle cell trait carriage and this irrespective of the parasite stage $(p>0.05)$. The level of infectivity of the mosquito was higher when feedings were performed on blood from HbAS genotypes compared to HbAA genotype blood, and the difference was even more significant when the blood pellet was resuspended with non-immune $A B$ plasma $(p<0.0001)$. No significant difference was observed in the infection complexity between HbAS and HbAA genotypes ( $\mathrm{p}>0.05)$.

Conclusion Plasmodium infection is not influenced by HbAS genotype regardless of parasite stage; the risk of anopheles infection is higher with blood from gametocyte carriers with sickle cell trait (HbAS). The sickle cell trait does not affect the multiplicity of infection.

\section{PO 8296 REDUCING LOSS TO FOLLOW-UP OF CHILDREN EXPOSED TO HIV IN THE PROVINCES OF MANICA AND SOFALA, CENTER OF MOZAMBIQUE}

Lúcia da Costa Vieira*, Arlete Mahumane. Beira Operational Research Center, Maputo, Mozambique

\subsection{6/bmjgh-2019-EDC.70}

Background Early childhood diagnosis of HIV is a challenge in many developing countries, including Mozambique. Approximately $50 \%$ of exposed children and HIV-positive are lost to follow-up, i.e. during Postpartum Consultation (CPP), at-risk child consultation (CCR) or ART consultation in the country. The objective was to carry out an intervention to reduce the loss to follow-up of children exposed to and positive for HIV in Manica and Sofala provinces.

Methods Intervention study in HIV-positive women and their children in CPP and CCR in six health facilities in 2016. Stepped-wedge design with 3 cohorts for 3 months of two health facilities randomly selected. Interventions included activist allocation, telephone calls to contact the mothers, guide the mothers with exposed child from CPP to CCR, active outreach to missed mothers, and initiation of ART in the CCR for 3 months. Data were collected from the health facilities and study books. Analysis was binomial logistic regression model with mixed effects.

Results Of the aggregated data, PCR + was 7.7\%, and proportion of HIV-positive women in CPP $17.4 \%$. In the control group only $24 \%$ of the mothers had more than 2 visits with 
us, compared with $60 \%$ in the intervention group $[\mathrm{OR}=2.05$ (95\% CI: 1.60, 2.62)]. In the intervention group, more children were transferred to CCR $52 \%$ vs $32 \%$ in the control $[\mathrm{OR}=1.7$ (CI: 1.3-2.41)], $65 \%$ of the mothers in intervention group reached at CCR vs $57 \%$ of the mothers in the control group $[\mathrm{OR}=1.69 \mathrm{CI}: 1.27-2.41)]$ and returned to receive the PCR result of their child, $6.7 \%$ in the control vs $8.2 \%$ in intervention $[\mathrm{OR}=2.3$ (CI: $1.36,3.87)]$.

Conclusion The intervention had a greater impact on the number of visits to CPP, the transfer of mothers from CPP to CCR, and the reception of PCR results in CCR by the companion.

\section{PO 8300 REGIONAL CENTER FOR REGULATORY EXCELLENCE IN CLINICAL TRIAL OVERSIGHT - TRAINING 2017}

Delese Darko*, Yvonne Adu-Boahen. Food and Drugs Authority, Accra, Ghana

\subsection{6/bmjgh-2019-EDC.71}

Background The competencies of the various national medicines regulatory agencies (NMRAs) in Africa vary which leads to generally porous regulatory systems for clinical trial oversight. Consequently, many trials have been conducted under unacceptable conditions compromising participants' safety and data credibility and resulted in questionable outcomes that are used for making scientific judgement in addressing issues of public health in Africa.

To improve the safety and quality of health technologies in Africa, the New Partnership for African Development (NEPAD) agency launched a programme to designate Regional Centres of Regulatory Excellence (RCOREs) with the specific objective of bridging existing gaps between African NMRAs through strengthening regulatory capacity of African Union member states. The Food and Drugs Authority (FDA), Ghana, was designated as RCORE for Clinical Trials oversight in May 2014.

Methods To achieve the RCORE objectives, the FDA collaborated with the School of Public Health (SPH), University of Ghana to develop a training manual and piloted a training programme with funds from the International AIDS Vaccine Initiative (IAVI) through NEPAD.

The programme, consisting of 4 compulsory modules, was organised from 6-30 November 2017 for 10 participants from Zambia, Sierra Leone, Liberia, Rwanda and Ghana. Interactive training methods in the form of theoretical and practical sessions were employed.

Results The pilot RCORE training was successful with expected training objectives achieved. Participants gained hands-on experience through activities like observing Good Clinical Practice inspection and a Technical Advisory Committee Meeting. Participants were given template tools to assist in developing regulatory guidelines and forms in their respective countries.

A follow-up questionnaire was circulated to participants to assess the impact of the training on their work. Feedback indicates that regulation of clinical trials has improved in their respective institutions.

Conclusion This pilot fellowship training was successful, leading to the improvement of clinical trial regulation in the participating countries.

\section{PO 8302 IMPACT OF TWO ANNUAL ROUNDS OF MASS DRUG ADMINISTRATION WITH DIHYDROARTEMISININ- PIPERAQUINE ON MALARIA TRANSMISSION IN A PROSPECTIVE COHORT STUDY}

2Julia Mwesigwa*, ${ }^{2}$ Jane Achan, ${ }^{2}$ Miriam Wathuo, ${ }^{2}$ Archibald Worwui, ${ }^{2}$ Nuredin Mohammed, ${ }^{2}$ Fatomatta Kanuteh, ' Jean-Pierre Van Geertruyden, ${ }^{2}$ Umberto D Alessandro. ${ }^{1}$ Faculty of Medicine and Health Sciences, University of Antwerp, Belgium; ${ }^{2}$ MRC Unit The Gambia at the London School of Hygiene and Tropical Medicine, Banjul, The Gambia

\subsection{6/bmjgh-2019-EDC.72}

Background Mass drug administration (MDA) may reduce malaria transmission in low-transmission areas and interrupt transmission. The impact of MDA with dihydroartemisininpiperaquine (DP) on malaria infection and clinical malaria was determined in a prospective cohort study in The Gambia.

Methods Single annual MDA rounds with DP were done in 2014 and 2015 in a prospective cohort among residents aged $>6$ months in twelve villages in The Gambia at the start of the transmission season in June. Monthly blood samples for microscopy and PCR were collected during the transmission season from July to December, post MDA and once before MDA during the dry season in April. The incidence of infection and clinical malaria post-MDA were compared to 2013 and mixed effects logistic regression models assessed the efficacy and risk of re-infection post MDA.

Results Coverage of 3 DP doses was $68.22 \%$ in 2014 and $65.60 \%$ in 2015 . Compliance to 3 doses was high, $83.11 \%$ in 2014 and $85.93 \%$ in 2015. Incidence of infection in 2014 (2014: IR=0.23 PPY, 2013: IR=1.12 PPY, $p<0.01$ ) and clinical malaria in 2014 (2014: IR=0.08 PPY, 2013: IR=0.39: $\mathrm{IRR}=0.22, \quad \mathrm{p}<0.01) \quad$ and $2015 \quad(2015: \quad \mathrm{IR}=0.19, \quad 2013$ : $\mathrm{IR}=0.38, \quad \mathrm{IRR}=0.50, \mathrm{p}<0.01$ ) was significantly lower after MDA compared to 2013. The incidence of clinical malaria remained higher in eastern Gambia compared to the western region. Subjects that took 3 DP doses had lower odds of infection in 2014 at 28 days $(\mathrm{OR}=0.61,95 \% \mathrm{CI}$ : $0.38-0.99)$ and 42 days (2014: $\mathrm{OR}=0.52$, 95\% CI: $0.29-0.89$ )

Conclusion A single annual MDA round with DP temporarily reduced malaria infection and clinical disease during the transmission season and subjects that took 3 doses had lower risk of infection. However, several MDA rounds covering the entire transmission season and some targeting the human reservoir during the dry season, are needed to achieve a more marked sustained reduction of transmission.

\section{PO 8313 PREDICTORS OF HEALTH INSTITUTIONAL READINESS FOR EBOLA EPIDEMIC CONTAINMENT IN NIGERIA: A STRUCTURAL EQUATION MODELLING (SEM) APPROACH}

${ }^{1}$ Joshua Adekeye*, ${ }^{1}$ Eustasius Musenge, ${ }^{2}$ Oyedunni Arulogun. ' University of the Witwatersrand, Johannesburg, South Africa; ${ }^{2}$ University of Ibadan, Ibadan, Nigeria

\subsection{6/bmjgh-2019-EDC.73}

Background There is paucity of literature on organisational readiness in the health space. Previous studies focus on epidemic preparedness and often depict readiness as a minor element in the implementation space. This study investigated the predictors of health institutional readiness to implement innovations for combating an Ebola epidemic outbreak in Nigeria. 